\title{
Utilization of Emergency Department Data for Drug Overdose Surveillance in North Carolina
}

\author{
Katherine J. Harmon ${ }^{\star 1}$, Scott Proescholdbell ${ }^{2}$, Steve Marshall ${ }^{1}$ and Anna Waller ${ }^{3}$ \\ ${ }^{1}$ Injury Prevention Research Center, Chapel Hill, NC, USA; ${ }^{2}$ North Carolina Division of Public Health, Raleigh, NC, USA; ${ }^{3}$ Carolina \\ Center for Health Informatics, Chapel Hill, NC, USA
}

\section{Objective}

To describe the epidemiologic characteristics for emergency department visits due to drug overdoses in North Carolina.

\section{Introduction}

In North Carolina there has been an escalation of poisoning deaths. In 2011 , the number of fatal poisonings was 1,368 deaths, with $91 \%$ classified as drug overdoses with the majority of those due to opioid analgesics.[1] Far greater numbers of drug overdoses result in hospitalization, emergency department (ED) or outpatient clinic visits, or resolve without the individual seeking medical attention. Although public health authorities have long employed death data for drug overdose surveillance in $\mathrm{NC}$, little attention has been paid to the use of ED data for this purpose. Through the North Carolina Disease Event Tracking and Epidemiologic Collection Tool (NC DETECT), NC collects information on $99.5 \%$ of all acute-care ED visits across the state, primarily for syndromic surveillance purposes. Despite the timeliness and completeness of this data system, drug overdose surveillance is a challenge due to lack of a standardized definition for the positive identification of opioid overdoses. In this study we used NC DETECT ED data to describe visits due to drug, and more specifically, opioid overdoses.

\section{Methods}

We performed a NC population-based study of all 2011 ED visits due to drug overdoses captured by the NC DETECT system using a surveillance definition based on the Centers for Disease Control and Prevention State Injury Indicators.[5] We identified ED visits as drug overdoses if the visit contained an International Classification of Diseases, 9th Edition Clinical Modification (ICD-9-CM) diagnosis code in the range of 960-979 (.0-.9) and/or an External Cause of Injury Code (E-code) in the range of E850-E858 (.0-.9), E950.0-E950.5, E962.0, or E980.0-E980.5 listed in any position. An ED visit was flagged as an opioid overdose if the visit contained a diagnosis code in the range of 965.0 and/or an E-code in the range of E850.0-E850.2. We generated summary statistics for all drug and opioid overdoses and looked at rates of ED visits by sex and age.

\section{Results}

In 2011, there were 35,193 ED visits due to poisonings; 22,992 of the ED visits were characterized as drug overdoses $(56.6 \%)$. The 2011 rate of ED visits due to drug overdoses was 238.1 ED visits per 100,000 person-years. We identified 3,251 ED visits as opioid overdoses with 312 visits due to heroin, 312 visits due to methadone, and 2,501 ED visits due to other or unspecified opioids. Slightly over one-half of these ED visits were among women (1,722 ED visits). The 2011 rate of ED visits due to opioid overdose was $33.7 \mathrm{ED}$ visits per 100,000 person-years. Among men, opioid overdose rates peaked at ages 20-24; among women, rates peaked at ages 45-54. Among opioid overdoses in which the intent was reported, $58.4 \%$ were unintentional, $24.7 \%$ were due to self-harm, and $15.2 \%$ were of undetermined intent. Patients presenting as an opioid overdose were likely to be admitted to the hospital, with $44.9 \%$ admitted to the hospital, $4.7 \%$ admitted to the intensive care unit (ICU), and $1.8 \%$ admitted to psychiatry; only $38.1 \%$ were discharged from the ED. Nearly one-half of ED visits due to opioid overdoses had an expected source of payment by the government, with $25.4 \%$ expected payment by Medicare, $21.1 \%$ by Medicaid, and $2.6 \%$ paid by some other governmental source. Another $26.2 \%$ of ED visits had an expected source of payment of self-pay.

\section{Conclusions}

The US is in the midst of a drug overdose epidemic, primarily due to opioid analgesics. Population-based data describing opioid overdoses in the ED is useful for describing the burden of these injuries and for the development of public health prevention strategies.

\section{Keywords}

Drug overdose; Public health surveillance; Emergency department data

\section{Acknowledgments}

These data were obtained from the NC DHHS/DPH NC DETECT system under a data use agreement. The NC DETECT Data Oversight Committee does not take responsibility for the scientific validity or accuracy of methodology, statistical analyses, results, or conclusions presented.

\section{References}

1.NC Injury and Violence Prevention Branch. Prescription and drug overdoses factsheet [Internet]. NC DPH, NC DHHS, Raleigh (NC): 2013 January [cited 2013 August 20]. Available from: http://injuryfreenc. ncdhhs.gov/About/PoisoningOverdoseFactSheet2013.pdf.

2.Thomas KE, Johnson RL. State injury indicators report: Instructions for preparing 2010 data [Internet]. NCIPC, CDC, Atlanta (GA): 2012 [cited September 5]. Available from http://www.cdc.gov/injury/ pdfs/2010 State_Injury_Indicator_Instructions-a.pdf.

\section{*Katherine J. Harmon}

E-mail: kjharmon@live.unc.edu 